\title{
EDDY FIELD MEASUREMENT BY USING SEARCH COILS FOR BENDING MAGNET
}

\author{
Y. Kanai* and M. Fujioka, Tohoku University, Sendai 980-8578, JAPAN \\ Y.Mori, M.Muto, E.Yanaoka, KEK-Tanashi, 3-2-1 Midori-cho, Tanashi 188-8501, JAPAN \\ H.Someya, F.Q. Zhang, T.Adachi, KEK, 1-1 Oho, Tsukuba 305-0801, JAPAN
}

\begin{abstract}
In order to estimate a field disturbance of a guiding field of a synchrotron due to an eddy current, magnetic fields in a bending magnet have been measured. The magnet was excited by a sinusoidal current with a repetition of $30 \mathrm{~Hz}$. Two search coils were used in the measurements. One is a reference coil and the other is a measurement coil. An imaginary part of an eddy field was derived from phase difference between two coil signals. The results were compared with a simple two-dimensional model.
\end{abstract}

\section{INTRODUCTION}

The JHF(Japan Hadron Facility) plan is being progressed. The JHF accelerator complex comprises the 200 $\mathrm{MeV}$ linac, the $3 \mathrm{GeV}$ synchrotron and the $50 \mathrm{GeV}$ synchrotron.

The $3 \mathrm{GeV}$ booster synchrotron is a rapid-cycling synchrotron with a repetition of $25 \mathrm{~Hz}$. In such a rapid-cycling synchrotron, it is expected that an eddy field disturbs a guiding field. There are some eddy current sources: metal wall of a vacuum chamber installed in a pulsed magnet and magnet components(e.g. magnet core, a end plate made of stainless steel and coil conductor).

Since a ceramic chamber is usually used in a rapidcycling synchrotron, the eddy current induced in magnet components mainly contributes to the field disturbance.

Therefore, it is important to estimate an effect of the eddy field due to magnet components. It has been known that the eddy field became larger as closer to the magnet edge [1].It implies that a contribution of the end plate to the eddy field is rather large than magnet core and conductor material. Taking into account this consideration, we constructed a simple two-dimensional model.

This paper describes the results of the field measurement and our simple model.

\section{FIELD MEASUREMENT}

\subsection{Measurement}

A test magnet for the JHF $50 \mathrm{GeV}$ ring was used for the eddy-field measurement. Fig. 1 shows the test magnet. The test magnet was excited with a repetition of $30 \mathrm{~Hz}$ by an ac power supply using IGBT [2]. Fig 2 shows cross sections of the magnet: the left is cut by $x-y$ plane and the right cut by $y-z$ plane. The coordinate system defined here is used through this paper.

\footnotetext{
*Email: ianak@tanashi.kek.jp
}

The field measurement was performed on the median $\operatorname{plan}(y=0)$.

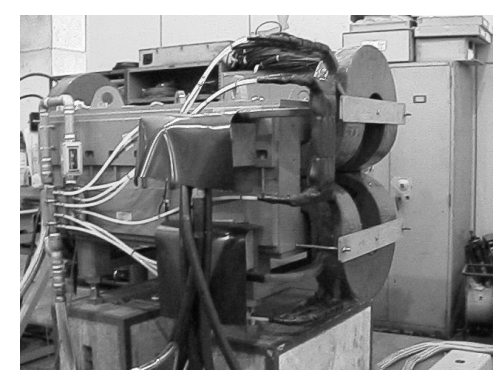

Figure 1: Test magnet for the JHF $50 \mathrm{GeV}$ ring

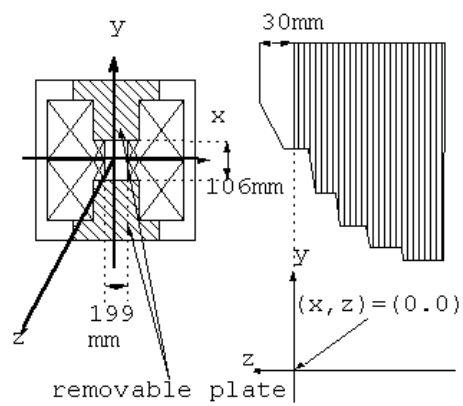

Figure 2: Cross section of the test magnet

In this measurement, two search coils were used: one is a reference coil and the other is a measurement coil. The reference coil was fixed at the center of the magnet, since the eddy current effect was expected to be negligible at this position. On the other hand, the measurement coil was moved on the median plan. Since the magnet is excited by a sinusoidal current with the repetition of $30 \mathrm{~Hz}$, magnetic flux density at the reference point $\left(B_{r e f}\right)$ is given by,

$$
B_{\text {ref }}=B_{0} e^{i \omega t}
$$

The flux density at measuring point $\left(B_{\text {meas }}\right)$ is given by,

$$
B_{\text {meas }}=\left(b_{1}+b_{2} e^{i \delta}\right) e^{i \omega t}=B_{1} e^{i(\omega t+\theta)}
$$

where $b_{1}$ is the amplitude of a main field and $b_{2} \exp (i \delta)$ is it the amplitude of the eddy field. $\theta$ and $B_{1}$ are given by,

$$
\theta=\tan ^{-1}\left(\frac{b_{2} \sin \delta}{b_{1}+b_{2} \cos \delta}\right)
$$

and

$$
\begin{aligned}
B_{1} & =\left(b_{1}{ }^{2}+b_{2}{ }^{2}+2 b_{1} b_{2} \cos \delta\right)^{\frac{1}{2}} \\
& \approx b_{1}+b_{2} \cos \delta
\end{aligned}
$$


respectively. Here, we assumed that the eddy field is sufficiently small compared with the main field, i.e. $b_{1} \gg b_{2}$

In terms of eqs (3) and (4), an imaginary part of the eddy field is obtained as follows:

$$
b_{2} \sin \delta=B_{1} \tan \theta
$$

In order to measure a contribution of the end plate definitely, we made a part of the end plate removable, as shown in figure 2.

Signals of two search coils were sent to a fft servo analyzer, and phase difference $\theta$ and $B_{1}$ was measured.

\subsection{Experimental Results}

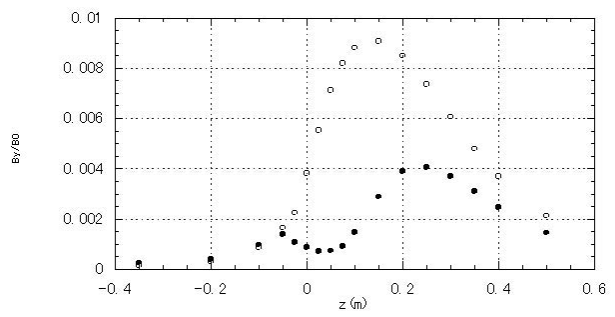

Figure 3: Longitudinal dependence of the eddy field.

The longitudinal dependence of the imaginary part of the eddy field is shown in figure 3 . Here, open-circles denote the results in the normal case and solid-circles denote the results in the case that the removable plate is changed by a Bakelite plate. The eddy field is normalized by a component of the main field $\left(B_{0}\right)$ at the magnet center.

The results show that the contribution of the end plate is rather large. It is consistent with the former results[1].

The contribution of the removable plate to the eddy field can be estimated by subtracting the latter results from the former. It will be compared with a simple two-dimensional model.

\section{TWO-DIMENSIONAL MODEL}

\subsection{Formulation}

The fundamental equations describing eddy currents are as follows:

$$
\begin{aligned}
\operatorname{rot} \vec{E} & =-\frac{\partial \vec{B}}{\partial t} \\
\operatorname{rot} \vec{H} & =\vec{j} \\
\vec{j} & =\sigma \vec{E},
\end{aligned}
$$

where $\vec{H}$ and $\vec{E}$ are the magnetic and electric field strength, respectively. $\vec{B}$ is the magnetic flux density and $\vec{j}$ is the current density, $\mu$ and $\sigma$ are magnetic permeability and electric conductivity, respectively.
It is assumed that all material parameters are constant both in time and space. Eliminating $\vec{E}$ and $\vec{j}$ from the fundamental equations, one can obtain the following equation.

$$
\nabla^{2} \vec{H}-\sigma \mu \frac{\partial \vec{H}}{\partial t}=0 .
$$

In a vacuum, assuming that $\sigma=0$,

$$
\nabla^{2} \vec{H}=0 .
$$

Here, the magnetic field $\vec{H}$ is superposition of the external field $\vec{H}_{\text {ext }}$ and the eddy field $\vec{v}$.

$$
\vec{H}=\vec{H}_{e x t}+\vec{v}
$$

In addition, setting $\vec{H}=\overrightarrow{\hat{H}} e^{i \omega t}$, we obtain the following equation:

$$
\nabla^{2} \overrightarrow{\hat{v}}-i \omega \sigma \mu\left(\overrightarrow{\hat{H}}_{e x t}+\overrightarrow{\hat{v}}\right)=0
$$

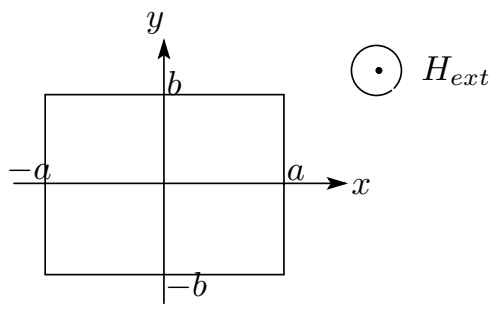

Figure 4: two-dimensional problem

In order to solve the above equation, we make a problem to be restricted to two-dimensional case, where the eddy current is bounded in a conducting plane, which is rectangular plane with size of $2 a \times 2 b$, and the external field is perpendicular to the plane, as shown figure 4.

The solution is obtained by giving a boundary condition that $\hat{v}=0$ along the circumference. The result is as follows:

$$
\begin{aligned}
\hat{v}= & -k \hat{H}_{e x t} \frac{16}{\pi^{2}} \sum_{m, n=1}^{\infty} \\
& \frac{(-1)^{m+n} \cos \frac{2 m-1}{2 a} \pi x \cos \frac{2 n-1}{2 b} \pi y}{\left\{\left(\frac{2 m-1}{2 a} \pi\right)^{2}+\left(\frac{2 n-1}{2 b} \pi\right)^{2}+k\right\}(2 m-1)(2 n-1)},
\end{aligned}
$$

where $k=i \omega \sigma \mu$ and we assumed that $\hat{H}_{\text {ext }}=$ constant. The eddy current can be obtained by $\vec{j}=\operatorname{rot} \overrightarrow{\hat{v}}$, therefore $j_{x}$ and $j_{y}$ is given by,

$$
\begin{aligned}
\frac{j_{x}}{\hat{H}_{e x t}} & =k \frac{16}{\pi^{2}} \sum_{m, n=1}^{\infty} \\
& \frac{(-1)^{m+n} \frac{2 n-1}{2 b} \pi \cos \frac{2 m-1}{2 a} \pi x \sin \frac{2 n-1}{2 b} \pi y}{\left\{\left(\frac{2 m-1}{2 a} \pi\right)^{2}+\left(\frac{2 n-1}{2 b} \pi\right)^{2}+k\right\}(2 m-1)(2 n-1)}
\end{aligned}
$$

and 


$$
\begin{aligned}
\frac{j_{y}}{\hat{H}_{e x t}} & =-k \frac{16}{\pi^{2}} \sum_{m, n=1}^{\infty} \\
& \frac{(-1)^{m+n} \frac{2 m-1}{2 a} \pi \sin \frac{2 m-1}{2 a} \pi x \cos \frac{2 n-1}{2 b} \pi y}{\left\{\left(\frac{2 m-1}{2 a} \pi\right)^{2}+\left(\frac{2 n-1}{2 b} \pi\right)^{2}+k\right\}(2 m-1)(2 n-1)} .
\end{aligned}
$$

A fraction of the eddy current of the conducting plane contributes the eddy field at the field point, $P$, by $d \vec{H}_{e d d y}$, as shown in figure 5 .

$$
d \vec{H}_{e d d y}=\frac{I}{4 \pi} \frac{d \vec{s}}{\left|\vec{r}_{1}\right|^{2}} \times \frac{\vec{r}_{1}}{\left|\vec{r}_{1}\right|} .
$$

We must also take into account magnetic charge, $\tau$, induced on the surface of the magnet core. $\tau$ is approximately given by

$$
\tau \approx \mu_{0}\left(\vec{H}_{e d d y}\right)_{\perp},
$$

where $\left(\vec{H}_{e d d y}\right)_{\perp}$ is the component of the eddy field perpendicular to the core surface. Hence, the contribution of the magnetic charge at $P, d \vec{H}_{m}$ is obtained as

$$
d \vec{H}_{m}=\frac{\tau d x^{\prime} d y^{\prime}}{4 \pi \mu_{0}\left|\vec{r}_{2}\right|^{2}} \frac{\vec{r}_{2}}{\left|\vec{r}_{2}\right|} .
$$

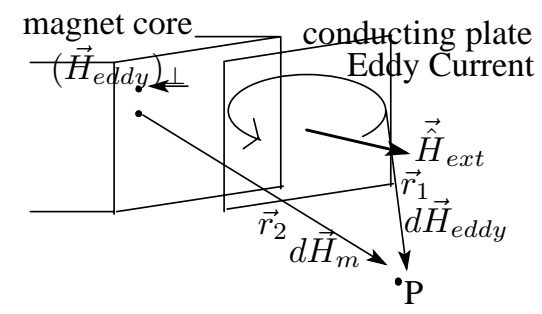

Figure 5: Model of the field calculation

Consequently, the magnetic field at $P$ is given by,

$$
\vec{H}=\int_{\text {coresurface }} d H_{m}+\int_{\text {conductorplane }} d H_{\text {eddy }}
$$

\subsection{Comparison with Measurements}

The figure 6 and 7 show longitudinal and transverse distribution of the eddy field, respectively. Open circles and dots denote experimental results and calculations, respectively.

Unfortunately, the values of the experimental results are larger than the calculation by a factor of 1.7 .

In order to compare a shape of distribution, each result is normalized by peak value of figure 7. As shown in the figures, the shape of the field distribution is well explained by our model.

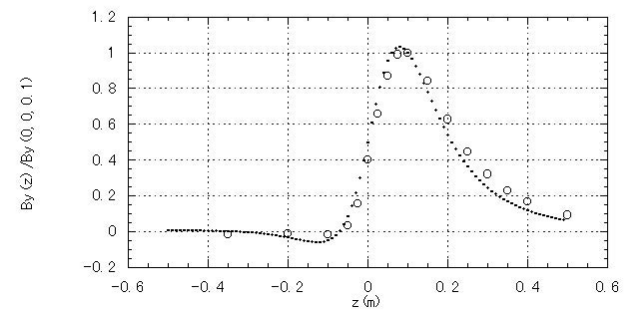

Figure 6: Longitudinal distribution of the eddy field

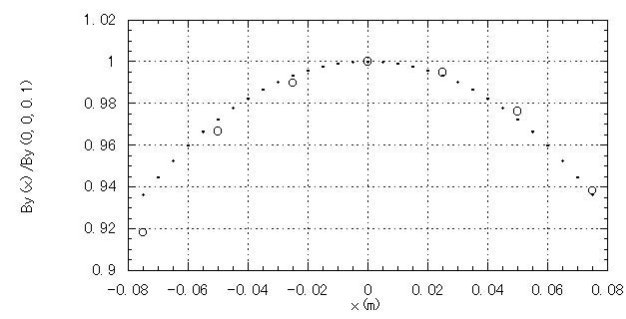

Figure 7: Transverse distribution of the eddy field

\section{CONCLUSION}

The field disturbance due to the eddy current induced in a magnet end plate was measured. The results are compared with a simple analytical model. The model can explain the shape of distribution, while the values are less than the experimental results. Such a discrepancy may be caused by an assumption of a constant external field $\left(\vec{H}_{e x t}\right)$, since $\vec{H}_{\text {ext }}$ is not constant actually on the end plate.

Another possibility is that the contribution of the magnetic charge induced by the eddy field is not sufficiently estimated. This problem is now being considered.

An eddy field due to a vacuum chamber in a pulsed magnet can be also calculated using our model. Therefore the eddy field due to the vacuum chamber will be measured soon.

\section{ACKNOWLEDGMENTS}

The authors would like to say thanks Dr. A. Takagi for his advice during this work. We also would like to thank KEK members for their fruitful help.

\section{REFERENCES}

[1] Y.Kanai, T.Adachi, M.Fujioka and Y.Mori, "Eddy field Measurement Using a Peaking-Strip", 11th Symposium on Accelerator Science and Technology, Japan,(1997).

[2] M.Muto, Y.Kanai, T.Kubo, Y,Mori, T.Morimoto, K.Niki, Y.Nishiyama, H,Sato, K.Shino and E.Yanaoka, "Magnets and Power Supply System of JHF 50-GeV Main Ring”,APAC'98,Japan,(1998). 